\title{
Vector boson fusion in the inert doublet model
}

\author{
Bhaskar Dutta* \\ Mitchell Institute for Fundamental Physics and Astronomy, Department of Physics and Astronomy, \\ Texas A\&M University, College Station, Texas 77843, USA \\ Guillermo Palacio ${ }^{\dagger}$ and Diego Restrepo \\ Instituto de Física, Universidad de Antioquia, \\ Calle 70 No. 52-21, Código Postal, 050010 Medellín, Colombia \\ José D. Ruiz-Álvarez ${ }^{\S}$ \\ Departamento de Física, Universidad de Los Andes, Código Postal, 111711 Bogotá, Colombia
}

(Received 4 December 2017; published 29 March 2018)

\begin{abstract}
In this paper we probe the inert Higgs doublet model at the LHC using vector boson fusion (VBF) search strategy. We optimize the selection cuts and investigate the parameter space of the model and we show that the VBF search has a better reach when compared with the monojet searches. We also investigate the Drell-Yan type cuts and show that they can be important for smaller charged Higgs masses. We determine the $3 \sigma$ reach for the parameter space using these optimized cuts for a luminosity of $3000 \mathrm{fb}^{-1}$.
\end{abstract}

DOI: $10.1103 /$ PhysRevD.97.055045

\section{INTRODUCTION}

The particle physics origin of $27 \%$ of the Universe is still unknown. The results from the direct, indirect detections and the collider experiment are investigating particle physics models which provide a dark matter candidate. The null results so far from these experiments have already ruled out many models. However, many models still exist with large regions of allowed parameter space. Since the LHC is ongoing, it will be crucial to come up with strategies to investigate the parameter spaces of these models to the maximum extent. In this paper, our main focus is to use the LHC searches to investigate one simple dark matter model by developing a search strategy and compare with the existing search strategies.

One of the simplest models which provides a dark matter (DM) candidate is the inert doublet model (IDM) [1,2], where an additional scalar doublet of $S U(2)_{L}$ odd under a global $Z_{2}$ is added to the Standard Model (SM). The lightest neutral component of the scalar doublet acts as a dark matter candidate. Recent accounts of the model are

\footnotetext{
*dutta@physics.tamu.edu

galberto.palacio@udea.edu.co

restrepo@udea.edu.co

§ose.ruiz@cern.ch
}

Published by the American Physical Society under the terms of the Creative Commons Attribution 4.0 International license. Further distribution of this work must maintain attribution to the author(s) and the published article's title, journal citation, and DOI. Funded by SCOAP ${ }^{3}$. given in [3-8], and further references are available therein. In particular, regarding the predictions of the model at colliders, the Drell-Yan (D-Y) production with lepton plus transverse missing energy signals at LHC have been extensively studied in the IDM as in [8-15]. We plan to investigate this model in this paper utilizing the vector boson fusion (VBF) search strategy. Since the dark matter candidate of this model has weak charges, $W, Z$ fusions are useful to produce these particles at the LHC. We will optimize the VBF cuts to improve the signal to the background ratio where the SM background mostly arises from $Z+$ jets. Utilizing the optimized cuts, we will show the reach of the LHC for the parameter space of the model in the ongoing and future runs. We will also compare this analysis with the existing search strategies.

We will investigate the LHC reach of the parameter space of the model without any restrictions arising from annihilation rate, direct and indirect detections since the correlations among these results to constrain the parameter space for the LHC require many assumptions. For example, the annihilation rate constraint arising from the DM content requires a knowledge of the history of the universe prior to big bang nucleosynthesis (BBN) which is unconstrained. The origin of the dark matter content, e.g., may be due to thermal, nonthermal [16-22], nonstandard cosmology (where the expansion rate can be different compared to the standard cosmology prior to the $\mathrm{BBN}$ ) [23-27]. Consequently, the annihilation rate corresponding to the $27 \%$ can be very different compared to the thermal dark matter in standard cosmology and in some nonthermal scenarios, the dark matter content may not be related to any 
annihilation rate at all. We, therefore, plan to search for this model at the LHC without showing any preference for a particular cosmological scenario. Further, there can be multiple DM candidates (e.g., axion and DM from the inert doublet model) and in such scenarios [28,29], the direct and indirect detection cross sections should be reduced by $R$ and $R^{2}$ respectively with $R \equiv \Omega h^{2} / 0.12$. From all these considerations, it appears that the search at the LHC should be strategized without applying restrictions arising from the thermal annihilation rate and the direct and indirect detection constraints. If the signals from a particular physics model which possess a DM candidate are discovered at the LHC, we would not only be able to establish that model but it also would give us an opportunity to investigate the cosmology in the pre-BBN era.

Following this strategy, the monojet final state has been used effectively to search for parameter space of this model in Ref. [4]. In this paper, we first optimize VBF cuts to search for the DM candidate in IDM. We will show that the VBF reach is better than the monojet final state. We will also show the parameter space where the D-Y type cuts can be important.

The paper is organized as follows. In Sec. II, we discuss the model. In Sec. III, we discuss the VBF signatures and develop VBF cuts. In Sec. IV, we discuss the parameter space reach for this model at the LHC and we conclude in Sec. V.

\section{THE MODEL}

The inert doublet model (IDM) is a minimal extension of the SM, where an additional $S U(2)_{L}$ scalar doublet, $\Phi$, odd under a $Z_{2}$ discrete symmetry is added. The lightest neutral component of the scalar doublet is the dark matter candidate.

The Lagrangian of the model is given by

$$
\mathcal{L}=\mathcal{L}_{\mathrm{SM}}+\left(D^{\mu} \Phi\right)^{\dagger} D_{\mu} \Phi-V(\Phi)
$$

where

$$
\begin{aligned}
V(\Phi)= & \mu_{2}^{2} \Phi^{\dagger} \Phi+\lambda_{2}\left(\Phi^{\dagger} \Phi\right)^{2}+\lambda_{3}\left(H^{\dagger} H\right)\left(\Phi^{\dagger} \Phi\right) \\
& +\lambda_{4}\left(H^{\dagger} \Phi\right)\left(\Phi^{\dagger} H\right)+\frac{\lambda_{5}}{2}\left[\left(\Phi^{\dagger} H\right)^{2}+\text { H.c. }\right] .
\end{aligned}
$$
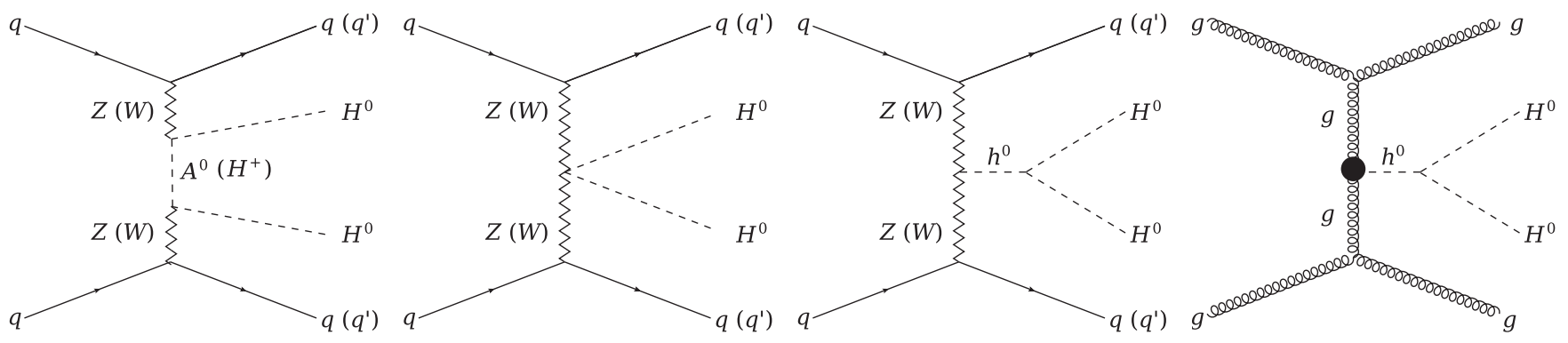

which controls the interactions through the Higgs portal. The components of the scalar doublets are defined as

$\Phi=\left(\begin{array}{c}H^{+} \\ \frac{1}{\sqrt{2}}\left(H^{0}+i A^{0}\right)\end{array}\right), \quad H=\left(\begin{array}{c}G^{+} \\ \frac{1}{\sqrt{2}}\left(v+h^{0}+i G^{0}\right)\end{array}\right)$.

The fields are written in the canonical form. $H^{0}, A^{0}$ and $H^{+}$ are the $C P$ even, $C P$ odd and charged scalar of the additional scalar doublet. $G^{+}$and $G^{0}$ are the Goldstone bosons of the $S U(2)_{L} \times U(1)_{Y}$ electroweak symmetry.

\section{SEARCH FOR VBF SIGNATURE AT THE LHC}

The IDM can be explored in the current and futures runs of the large hadron collider (LHC) with a center of mass energy $\sqrt{s}=13 \mathrm{TeV}$. Since the DM candidate $H_{0}$ has weak charges, it can be produced via a VBF strategy [30] at the LHC. The VBF search for the DM investigates missing energy in the association of two high $E_{T}$ jets with large $|\Delta \eta|$ between the jets in the final state.

The important contributions to the VBF total cross section for this model are displayed in Fig. 1. The last two diagrams are only important for large values of $\lambda_{L}$, while the first two diagrams can have large destructive interferences for small values of $\lambda_{L}$ and not too large splitting between the set of inert scalar masses. The blob in the gluon diagram denotes the effective coupling between the gluons and the SM Higgs.

The inclusive cross section for the process $p p \rightarrow$ $H^{0} H^{0} j j$ for a fixed dark matter mass of $150 \mathrm{GeV}$ is displayed in Fig. 2 as a function of $\lambda_{L}$ for several values of $M_{A^{0}}=M_{H^{+}}$. Note that for specific values of $M_{A^{0}}=M_{H^{+}}$, the Drell-Yan production of inert scalars for small $\lambda_{L}$ can be enhanced, because of resonant production of gauge bosons which give rise to the two jets.

FIG. 1. Feynman diagrams which contribute to $p p \rightarrow H^{0} H^{0} j j$ in the IDM. 


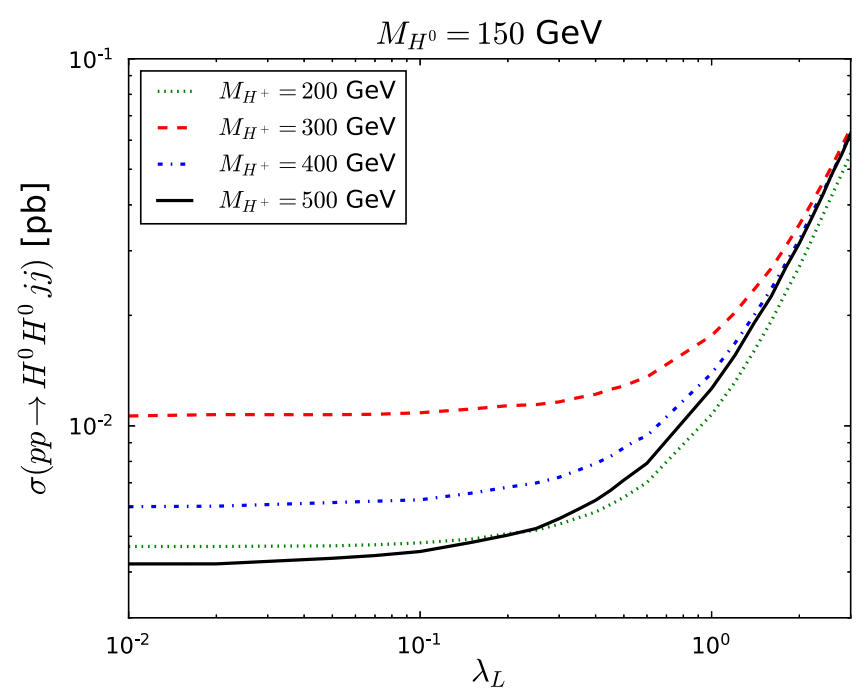

FIG. 2. Cross section for the process $p p \rightarrow H^{0} H^{0} j j$ as a function of $\lambda_{L}$ for $M_{H^{0}}=150 \mathrm{GeV}$, and several values of $M_{A^{0}}=M_{H^{+}}$.

The VBF topology relies on a set of characteristics of the events from the point of view of a detector as in the ATLAS or CMS experiments. The two jets produced from such a signature are located in different hemispheres of the detector, which means that $\eta\left(j_{1}\right) \times \eta\left(j_{2}\right)<0$. Additionally, these two jets are also well separated in the pseudorapidity. We expect then that the two jets from backgrounds faking the VBF topology have smaller separation in $\eta$ than the two jets from our signal. Finally, a key characteristic of the two jets from the VBF topology is that their invariant mass is larger than for a couple of non-VBF jets. Some contributions to the VBF signal have been considered in [31] which does not include some of the essential VBF cuts, e.g., large $|\Delta \eta|$, large $M_{j_{1} j_{2}}$, etc. Our cuts originate from the experimental searches as mentioned above [32,33] which we optimize further. Consequently, we obtain a much larger reach for the parameter space.

All these VBF characteristics significantly reduce backgrounds and give us a different set of background than monojet searches. Our main backgrounds are $Z \rightarrow \nu \bar{\nu}+$ jets and $W^{+/-} \rightarrow l \bar{\nu}+$ jets, where the lepton is missed by the detector reconstruction (for example if it is produced outside the experiment acceptance or fails isolation criteria). $Z \rightarrow \nu \bar{\nu}+$ jets background will be referred to in the following as simply $Z+$ jets. The QCD contribution to our background expectations is very small, and we consider it negligible for simplicity of this work.

To design our analysis we have used MC simulations of $Z+$ jets and the signal. We have used MADGraPH [34] to simulate the partonic process while PYTHIA 8 [35] for the hadronization and showering. Finally, we have processed our samples with DeLPHES [36] to simulate a detector response. We have used default configurations from the packages and we have worked specifically with the CMS

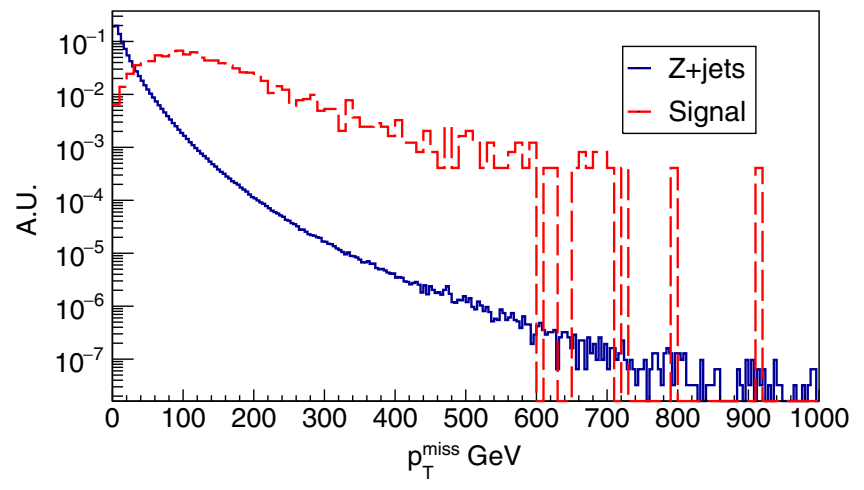

FIG. 3. $p_{T}^{\text {miss }}$ signal and $Z+$ jets background distributions before analysis selection. Both distributions have been normalized to unity.

experiment simulation done by DELPHES. We do not expect significant differences to our conclusions by switching the detector simulation to an ATLAS-like configuration. We have used AK4 (Anti-kt algorithm with $R=0.4$ ) jets that are reconstructed with the FASTJET package [37]. Jets were reconstructed in a rapidity acceptance of $|\eta(j)|<5$. For the simulation of the signal we have used the IDM model implementation [11,38] available in the FEYNRULES [39] models database.

Based on the analysis presented in [32], we assume that the $W^{+/-}+$jets background is kinematically similar to the $Z+$ jets background. and that after full selection our background contribution will be composed $70 \%$ by $Z$-jets events and $30 \%$ by $W^{+-}+$jets. Moreover, as we just consider the significance,

$$
\sigma \equiv \frac{S}{\sqrt{S+B}}
$$

as a figure of merit over the total number of events to determine the goodness of the selection, we are not affected by potential small kinematic differences between our two main backgrounds.

For the analysis we propose we relied on the following set of variables:

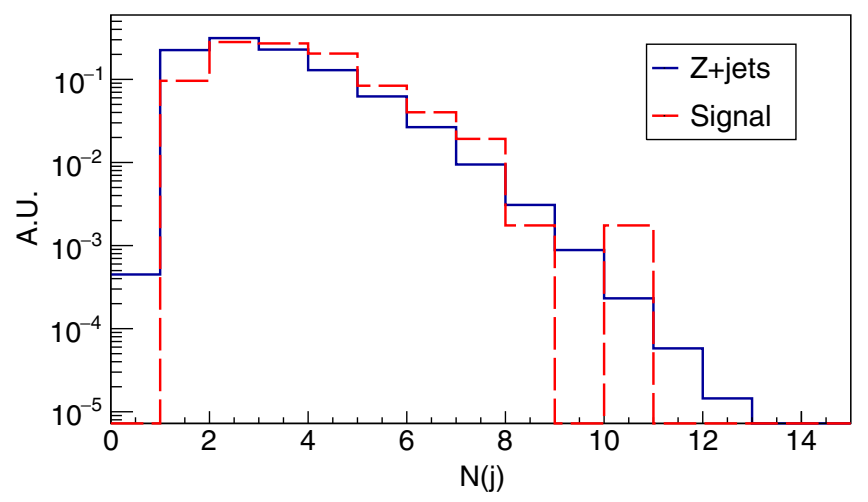

FIG. 4. $\quad N(j)$ signal and $Z+$ jets background distributions after cut 1 . Both distributions have been normalized to unity. 

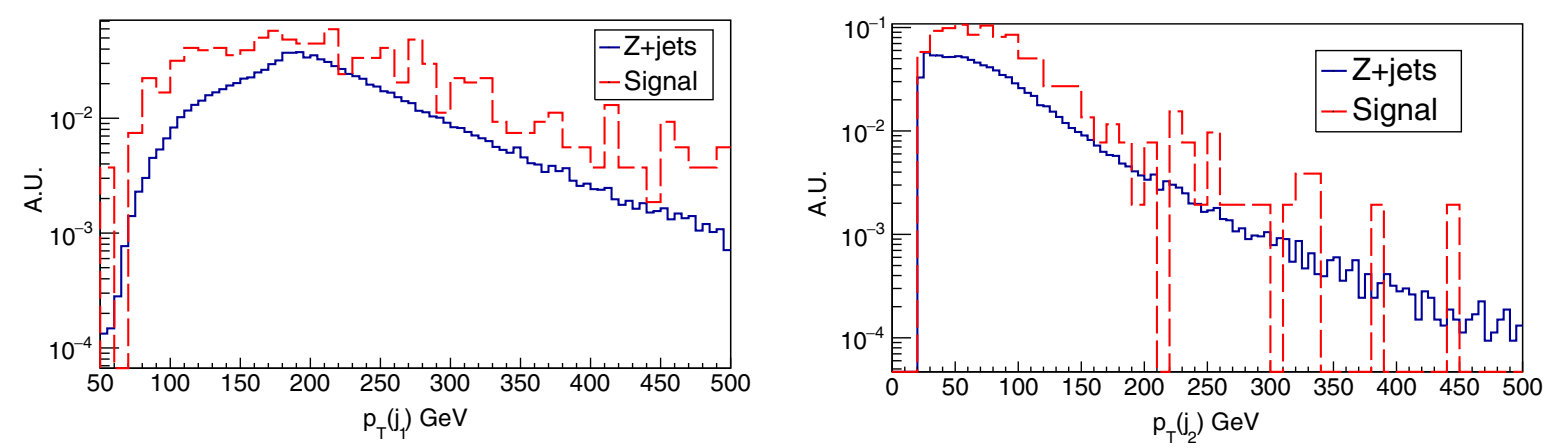

FIG. 5. $\quad p_{T}\left(j_{1}\right)$ (left) and $p_{T}\left(j_{2}\right)$ (right) signal and $Z+$ jets background distributions before analysis selection. Both distributions have been normalized to unity.

(i) $p_{T}^{\text {miss }}=-\left|\sum_{i=0}^{N(j)} \vec{p}_{T}\left(j_{i}\right)\right|$ denoted in the literature as transverse missing energy.

(ii) $N(j)$ the jet multiplicity.

(iii) The two leading jets $p_{T}, p_{T}\left(j_{1}\right)$ and $p_{T}\left(j_{2}\right)$.

(iv) $\eta\left(j_{1}\right) \times \eta\left(j_{2}\right)$.

(v) $\left|\Delta \eta\left(j_{1}, j_{2}\right)\right|$.

(vi) $M\left(j_{1}, j_{2}\right)$, the invariant mass of the two leading jets.

The selection was followed having the greatest significance in the order of the variables that have been cited. $N(j)$ was fixed to be greater than 1 and $\eta\left(j_{1}\right) \times \eta\left(j_{2}\right)<0$. The signal samples used for the optimization was produced with $M_{H^{0}}=65 \mathrm{GeV}, M_{H^{+}}=M_{A^{0}}=750 \mathrm{GeV}$ and $\lambda_{L}=$ 0.2 , but it has been checked that changing the $\lambda_{L}$ parameter we do not gain reoptimizing the selection.

The set of cuts that drives the analysis to the greatest significance is the following:

Cut 1: $p_{T}^{\text {miss }}>180 \mathrm{GeV}$

Cut 2: $N(j) \geq 2$

Cut 3: $p_{T}\left(j_{1}\right)>100 \mathrm{GeV}$

Cut 4: $p_{T}\left(j_{2}\right)>50 \mathrm{GeV}$

Cut 5: $\eta\left(j_{1}\right) \times \eta\left(j_{2}\right)<0$

Cut 6: $\left|\Delta \eta\left(j_{1}, j_{2}\right)\right|>4.2$

Cut 7: $M\left(j_{1}, j_{2}\right)>1 \mathrm{TeV}$

The selection on the missing energy was chosen quite high because this variable is normally used for the trigger in the experiments for dark matter searches. It would be a good improvement for this search if this threshold could be lowered down as much as possible in the triggers used by the experiments. A comparison between the signal and the main background on this variable before any cut can be found in Fig. 3. It can be seen that our signal is expected to have greater missing transverse momentum than the main background.

In Fig. 4 the jet multiplicity of our signal and main background after cut 1 is shown. Figure 5 shows the leading jets transverse momentum at the same stage of the selection and for the same samples. From the jets $p_{T}$ distributions it can be seen that however the main background tends to have a quite energetic first leading jet and a gain in sensitivity can be achieved cutting the events at the lower tail of the distribution. For the subleading jet, the signal shows clearly more energetic jets than the main background.

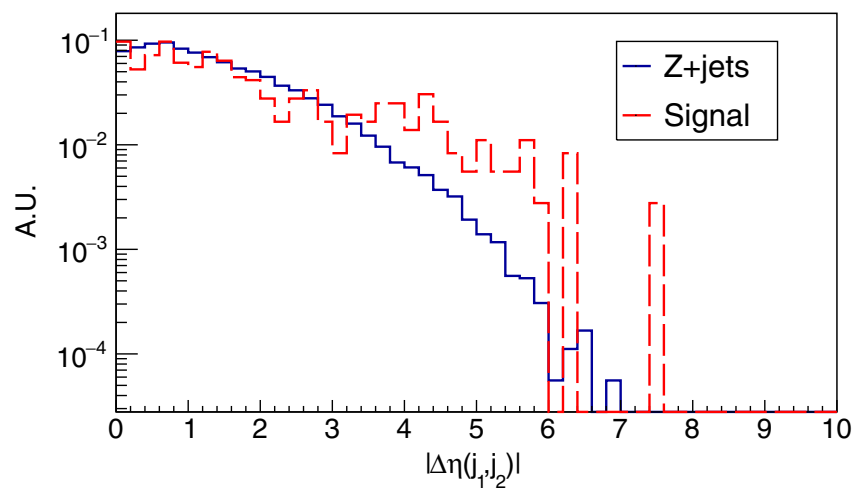

FIG. 6. $\Delta \eta\left(j_{1}, j_{2}\right)$ signal and $Z+$ jets background distributions after cut 4 . Both distributions have been normalized to unity.

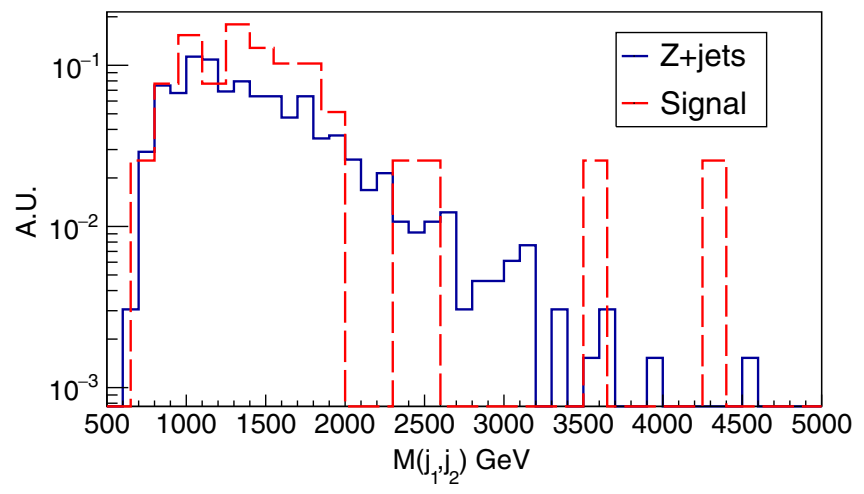

FIG. 7. $\quad M\left(j_{1}, j_{2}\right)$ signal and $Z+$ jets background distributions after cut 6 . Both distributions have been normalized to unity.

The pseudorapidity separation of the two leading jets is shown in Fig. 6 after cut 4 . We can corroborate from all of these cited distributions that the signal tends to have the greatest separation between the two leading jets than the main background.

Finally, further differences between the signal and the main background can be found in the invariant mass of the two leading jets. The distribution for signal and $Z+$ jets background after cut 6 are shown in Fig. 7.

The efficiencies of each cut are displayed in Table I. 
TABLE I. Efficiencies for signal and $Z+$ jets background for different stages of the selection.

\begin{tabular}{|c|c|c|c|c|}
\hline \multirow[b]{2}{*}{ Process } & \multicolumn{2}{|c|}{ Efficiency per cut } & \multicolumn{2}{|c|}{ Cumulative efficiency } \\
\hline & Signal & $Z+$ jets & Signal & $Z+$ jets \\
\hline Initial number of $\mathrm{MC}$ events & 2447 & 30996944 & 2447 & 30996944 \\
\hline Cut 1 & $(23.38 \pm 0.86) \%$ & $\left(0.22 \pm 8 \times 10^{-4}\right) \%$ & $(23.38 \pm 0.86) \%$ & $\left(0.22 \pm 8 \times 10^{-4}\right) \%$ \\
\hline Cut 4 & $(63.11 \pm 2.02) \%$ & $(51.95 \pm 0.19) \%$ & $(14.75 \pm 0.72) \%$ & $\left(0.12 \pm 6 \times 10^{-4}\right) \%$ \\
\hline Cut 6 & $(10.80 \pm 1.63) \%$ & $(1.82 \pm 0.07) \%$ & $(1.59 \pm 0.25) \%$ & $\left(2.11 \times 10^{-3} \pm 8.3 \times 10^{-5}\right) \%$ \\
\hline Cut 7 & $(84.62 \pm 5.78) \%$ & $(82.57 \pm 1.48) \%$ & $(1.35 \pm 0.23) \%$ & $\left(1.74 \times 10^{-3} \pm 7.5 \times 10^{-5}\right) \%$ \\
\hline
\end{tabular}

In Table I we have cited the efficiencies of the selection for a signal with $\lambda_{L}=0.2$. These efficiencies actually have a dependence on this parameter. When $\lambda_{L}$ is greater than 1 , the first diagram displayed in Fig. 1 becomes subdominant, and therefore changing the selection efficiency. Therefore we have scanned $\lambda_{L}$ between 0.01 and 10 , and we found efficiencies between $1 \%$ and $5 \%$. The efficiencies obtained from this scan have been used in the results section to calculate the significance as a function of $\lambda_{L}$. From Table I we can see that cut 7 is not resulting in a strong increase in the selection discrimination power. However, this cut, inspired from experimental results [32,33], would be more discriminant with a different technique to estimate backgrounds as with data-driven methods.

\section{RESULTS}

Using the cuts developed in Table I, we show the significance as a function of $M_{H_{0}}$ in Figs. 8 and 9 for 30 and $3000 \mathrm{fb}^{-1}$ luminosities and two different values of $\lambda$. In Table I we have quoted only statistical uncertainties, however in our results we have considered a higher uncertainty of $30 \%$ over signal and background yields to have a more realistic approach including other sources of uncertainties (e.g., PDF, cross section, ...). We find that larger values of $M_{H^{ \pm}}$and $\lambda_{L}$ produce larger significance for $\mathrm{VBF}$ analysis due to large production cross sections.

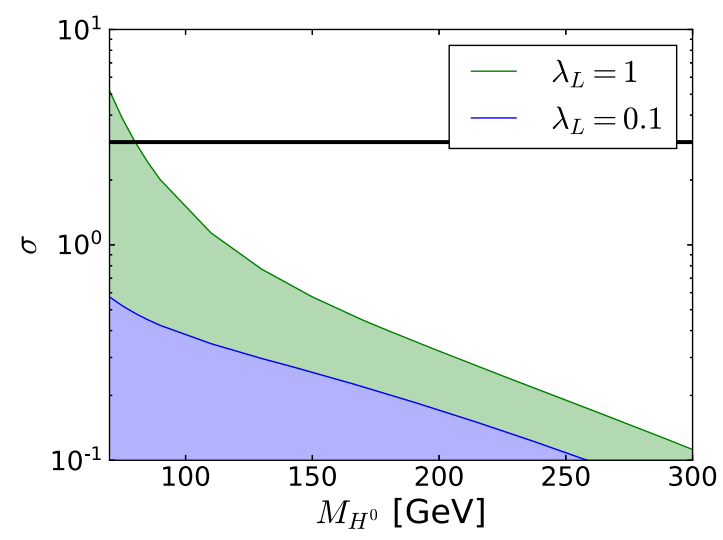

For $\lambda_{L} \sim 1$, the $3 \sigma$ reach can go up to $M_{H^{0}} \sim 200 \mathrm{GeV}$ for $3000 \mathrm{fb}^{-1}$ luminosity.

In Fig. 10, we show the $3 \sigma$ reach of our VBF cuts in the $\lambda_{L}$ and $M_{H^{0}}$ parameter space for various luminosities ranging from 30 to $3000 \mathrm{fb}^{-1}$. We maximized the production cross sections by choosing $M_{H^{ \pm}}$appropriately. We can see that the reach for the parameter space is substantial. The $3 \sigma$ reach of the parameter space can go up to $280 \mathrm{GeV}$ of $M_{H^{0}}$ for larger values of $\lambda_{L}$. For $\lambda_{L} \sim 10^{-2}$, the $3 \sigma$ reach for the DM mass goes up to $M_{H^{0}} \sim 125 \mathrm{GeV}$.

The IDM can be constrained using monojet-type searches at the LHC. However the reach of the VBF search is better than the monojet search. In Table II, we compare the significance of the monojet search using the cuts as given in $[4,40]$ with the VBF search strategy as developed in this paper at $20 \mathrm{fb}^{-1}$. In the table, $S_{\mathrm{MJ}, \mathrm{VBF}}, B_{\mathrm{MJ}, \mathrm{VBF}}$ and $\sigma_{\mathrm{MJ}, \mathrm{VBF}}$ are signal, background events and significances for monojet and $\mathrm{VBF}$ selection cuts respectively where we find that the VBF searches are more effective in probing the IDM models.

If $M_{H^{+}}$is not so large $(<500 \mathrm{GeV}), H^{+}$can be produced efficiently with a larger cross section which then subsequently decays into $H^{0}$. In such parameter space, Drell-Yan type cuts can be useful. In Table III, we show some Benchmark points where we vary $H^{+}, H^{0}$ masses and $\lambda_{L}$ to see effects on $\sigma\left(p p \rightarrow H^{0} H^{0} j j\right)$ where we find that if

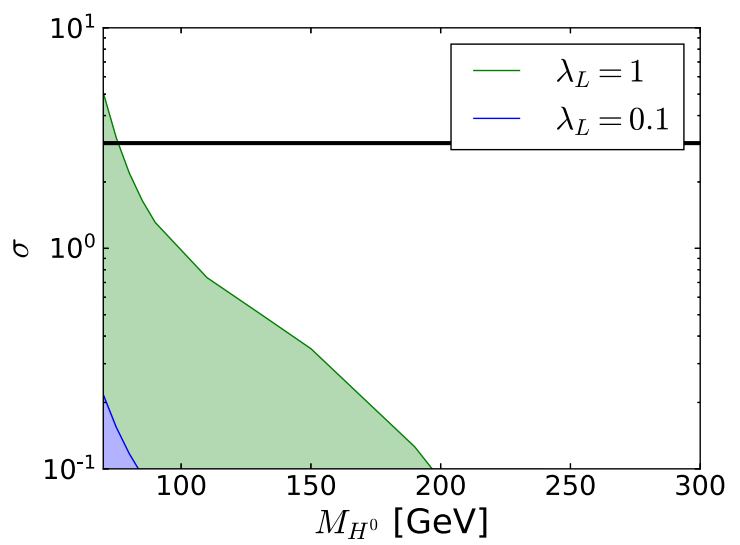

FIG. 8. Significance for DM candidate discovery for a luminosity of $30 \mathrm{fb}^{-1}$. The charged scalar mass is $750 \mathrm{GeV}(250 \mathrm{GeV})$ in the left (right) panel. 

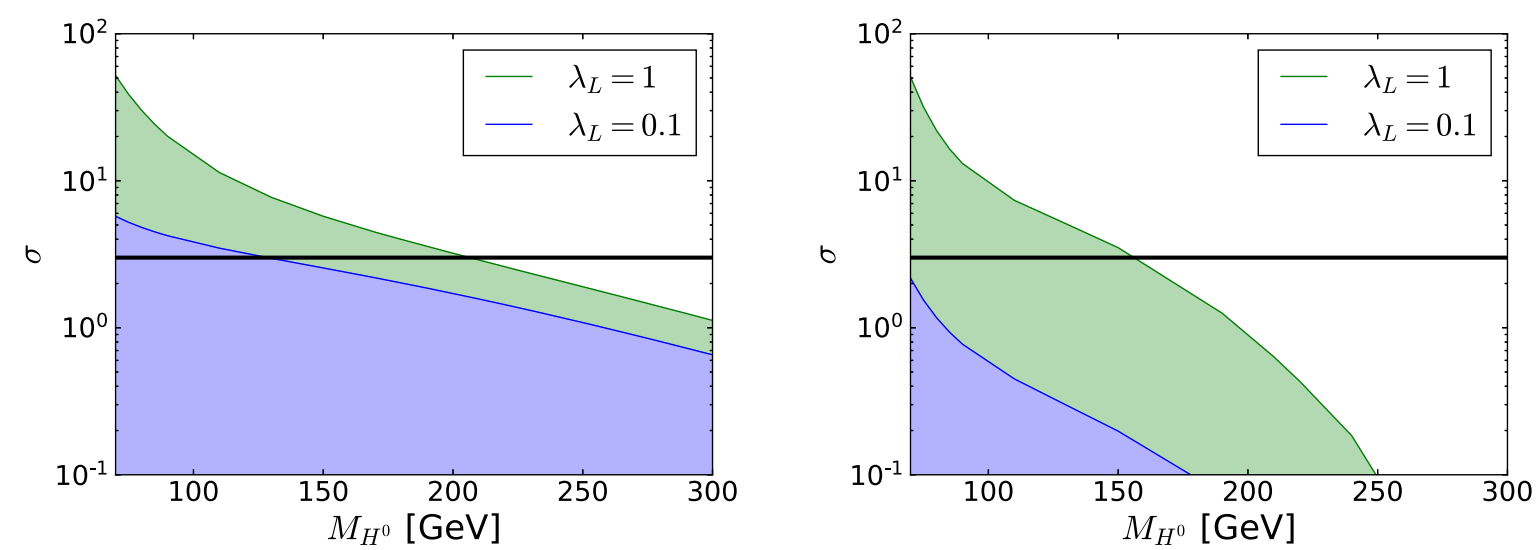

FIG. 9. Significance for DM candidate discovery for a luminosity of $3000 \mathrm{fb}^{-1}$. The charged scalar mass is $750 \mathrm{GeV}(250 \mathrm{GeV})$ in the left (right) panel.

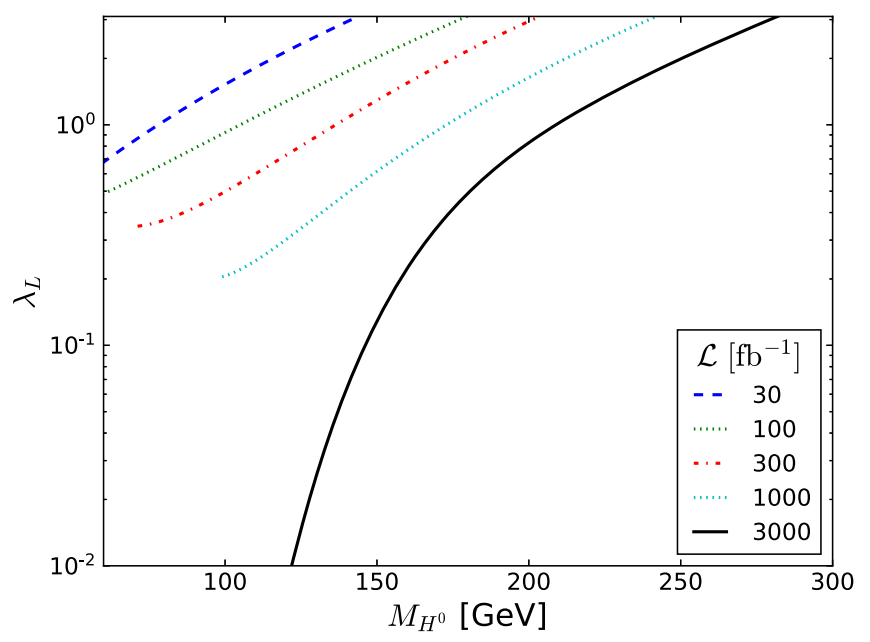

FIG. 10. $3 \sigma$ reach for $\lambda_{L}$ vs $M_{H^{0}}$ parameter space for various luminosities in $\mathrm{fb}^{-1}$.

the charged Higgs mass is smaller, then the cross section gets larger.

We then apply VBF and D-Y type cuts on all the scenarios where the D-Y type cuts are defined in [31]:

TABLE II. Sensitivities for $M_{H^{+}}=M_{A^{0}}=750 \mathrm{GeV}$ and $M_{H_{0}}=110 \mathrm{GeV}$ for several $\lambda_{L}$ values, with an integrated luminosity of $20 \mathrm{fb}^{-1}$.

\begin{tabular}{lccc}
\hline \hline$\lambda_{L}$ & $S_{\mathrm{MJ}}$ & $B_{\mathrm{MJ}}$ & $\sigma_{\mathrm{MJ}}$ \\
\hline 0.01 & 3 & & 0.015 \\
0.1 & 3.7 & 134044 & 0.02 \\
1.0 & 31 & & 0.15 \\
\hline \hline$\lambda_{L}$ & $S_{\mathrm{VBF}}$ & $B_{\mathrm{VBF}}$ & $\sigma_{\mathrm{VBF}}$ \\
\hline 0.01 & 4.6 & & 0.21 \\
0.1 & 7.5 & 476 & 0.35 \\
1.0 & 25 & & 1.1 \\
\hline \hline
\end{tabular}

$N(j)=2, N(b)=0, N(l)=0, p_{T}^{\text {miss }}>260 \mathrm{GeV}, p_{T}\left(j_{1}\right)>$ $120 \mathrm{GeV}, p_{T}\left(j_{2}\right)>90 \mathrm{GeV}, 75 \mathrm{GeV} \leq M_{j_{1} j_{2}} \leq 90 \mathrm{GeV}$, $\Delta R_{j_{1} j_{2}}<1.8$. We show our results in Table IV where we compare these cuts. In the table, $S_{\mathrm{DY}, \mathrm{VBF}}, B_{\mathrm{DY}, \mathrm{VBF}}$ and $\sigma_{\mathrm{DY}, \mathrm{VBF}}$ are signal, background events and significances for $\mathrm{D}-\mathrm{Y}$ and VBF selection cuts, respectively. We find that for $M_{H^{+}} \geq 500 \mathrm{GeV}$, the significance becomes much better for $\mathrm{VBF}$ analysis.

TABLE III. Benchmark scenarios for the comparison.

\begin{tabular}{lccccc}
\hline \hline & $\begin{array}{c}m_{H^{+}} \\
{[\mathrm{GeV}]}\end{array}$ & $\begin{array}{c}m_{A^{0}} \\
{[\mathrm{GeV}]}\end{array}$ & $\begin{array}{c}m_{H^{0}} \\
{[\mathrm{GeV}]}\end{array}$ & \multicolumn{1}{c}{$\begin{array}{c}\lambda_{L} \\
\text { Benchmark }\end{array}$} & $\begin{array}{c}\left.\sigma p \rightarrow H^{0} H^{0} j j\right) \\
{[\mathrm{pb}]}\end{array}$ \\
\hline BP1 & 200 & 189.5 & 65 & 0.009 & 0.067 \\
BP2 & 500 & 494 & 65 & 0.009 & 0.009 \\
BP3 & 750 & 750 & 65 & 0.009 & 0.008 \\
BP4 & 750 & 750 & 110 & 0.009 & 0.005 \\
BP5 & 750 & 750 & 65 & 0.500 & 0.274 \\
\hline \hline
\end{tabular}

TABLE IV. Comparison between our analysis and the one implemented in Ref. [31], with an integrated luminosity of $1000 \mathrm{fb}^{-1}$.

\begin{tabular}{lrrr}
\hline \hline Benchmark & $B_{\mathrm{DY}}$ & $S_{\mathrm{DY}}$ & $\sigma_{\mathrm{DY}}$ \\
\hline BP1 & & 148 & 1.59 \\
BP2 & \multirow{2}{*}{8500} & 68 & 0.73 \\
BP3 & & 15 & 0.16 \\
BP4 & & 85 & 0.11 \\
BP5 & & $S_{\mathrm{VBF}}$ & 0.92 \\
\hline \hline Benchmark & $B_{\mathrm{VBF}}$ & 38 & $\sigma_{\mathrm{VBF}}$ \\
\hline BP1 & & 198 & 0.25 \\
BP2 & & 309 & 1.28 \\
BP3 & 23809 & 3579 & 1.99 \\
BP4 & & 1.51 \\
BP5 & & 21.6 \\
\hline \hline
\end{tabular}




\section{CONCLUSIONS}

In this paper we utilize VBF search strategy to probe the parameter space of the inert doublet model where $H_{0}$ is a dark matter candidate. We probe the parameter space of the model at the LHC without applying constraints from the annihilation rate and the data from the direct and indirect experiments, since these constraints can be relaxed in various cosmological scenarios.

The VBF search is centered around the requirement of two high $p_{T}$ jets which located in different hemispheres of the detector, $\eta\left(j_{1}\right) \times \eta\left(j_{2}\right)<0$, which are also well separated in the pseudorapidity and their invariant mass is larger than for a couple of non-VBF jets. We further optimize the VBF cuts and found that $p_{T}^{\text {miss }}>180 \mathrm{GeV}$, $p_{T}\left(j_{1(2)}\right)>100(50) \mathrm{GeV},|\Delta \eta|>4.2, M\left(j_{1}, j_{2}\right)>1 \mathrm{TeV}$, $N(j) \geq 2, \eta\left(j_{1}\right) \times \eta\left(j_{2}\right)<0$ provide the largest significance. The dominant background arises from $Z+$ jets and we showed the signal and background distributions after each of these cuts. We showed that the VBF search has a better reach when compared with the monojet searches. We also showed that for $M_{H^{+}}<500 \mathrm{GeV}$, the D-Y type of cuts provide better significances while the VBF cut works much better for heavier $M_{H^{+}}$. We showed the $3 \sigma$ reach of the $\lambda_{L}-M_{H^{0}}$ parameter space of the model using VBF cuts for various luminosities and found that the reach can go up to $280 \mathrm{GeV}$ of Higgs mass for a luminosity of $3000 \mathrm{fb}^{-1}$.

\section{ACKNOWLEDGMENTS}

B. D. acknowledges support from DOE Grant No. desc0010813. D. R. acknowledges support from the Grants Sostenibilidad-GFIF and CODI-IN650CE, and COLCIENCIAS through the Grant No. 111-565-84269. J. D. Ruiz-Álvarez gratefully acknowledges the support of COLCIENCIAS, the Administrative Department of Science, Technology and Innovation of Colombia.
[1] N. G. Deshpande and E. Ma, Pattern of symmetry breaking with two Higgs doublets, Phys. Rev. D 18, 2574 (1978).

[2] R. Barbieri, L. J. Hall, and V.S. Rychkov, Improved naturalness with a heavy Higgs: An alternative road to LHC physics, Phys. Rev. D 74, 015007 (2006).

[3] B. Eiteneuer, A. Goudelis, and J. Heisig, The inert doublet model in the light of Fermi-LAT gamma-ray data: A global fit analysis, Eur. Phys. J. C 77, 624 (2017).

[4] A. Belyaev, G. Cacciapaglia, I. P. Ivanov, F. Rojas, and M. Thomas, Anatomy of the inert two Higgs doublet model in the light of the LHC and non-LHC dark matter searches, Phys. Rev. D 97, 035011 (2018).

[5] F. von der Pahlen, G. Palacio, D. Restrepo, and O. Zapata, Radiative type III seesaw model and its collider phenomenology, Phys. Rev. D 94, 033005 (2016).

[6] C. Garcia-Cely, M. Gustafsson, and A. Ibarra, Probing the inert doublet dark matter model with Cherenkov telescopes, J. Cosmol. Astropart. Phys. 02 (2016) 043.

[7] F. S. Queiroz and C. E. Yaguna, The CTA aims at the inert doublet model, J. Cosmol. Astropart. Phys. 02 (2016) 038.

[8] N. Blinov, J. Kozaczuk, D. E. Morrissey, and A. de la Puente, Compressing the inert doublet model, Phys. Rev. D 93, 035020 (2016).

[9] M. Hashemi and S. Najjari, Observability of inert scalars at the LHC, Eur. Phys. J. C 77, 592 (2017).

[10] A. Datta, N. Ganguly, N. Khan, and S. Rakshit, Exploring collider signatures of the inert Higgs doublet model, Phys. Rev. D 95, 015017 (2017).

[11] G. Belanger, B. Dumont, A. Goudelis, B. Herrmann, S. Kraml, and D. Sengupta, Dilepton constraints in the inert doublet model from run 1 of the LHC, Phys. Rev. D 91, 115011 (2015).
[12] M. Gustafsson, S. Rydbeck, L. Lopez-Honorez, and E. Lundstrom, Status of the inert doublet model and the role of multileptons at the LHC, Phys. Rev. D 86, 075019 (2012).

[13] X. Miao, S. Su, and B. Thomas, Trilepton signals in the inert doublet model, Phys. Rev. D 82, 035009 (2010).

[14] E. Dolle, X. Miao, S. Su, and B. Thomas, Dilepton signals in the inert doublet model, Phys. Rev. D 81, 035003 (2010).

[15] Q.-H. Cao, E. Ma, and G. Rajasekaran, Observing the dark scalar doublet and its impact on the standard-model Higgs boson at colliders, Phys. Rev. D 76, 095011 (2007).

[16] J. D. Barrow, Massive particles as a probe of the early universe, Nucl. Phys. B208, 501 (1982).

[17] M. Kamionkowski and M. S. Turner, Thermal relics: Do we know their abundances?, Phys. Rev. D 42, 3310 (1990).

[18] T. Moroi and L. Randall, Wino cold dark matter from anomaly mediated SUSY breaking, Nucl. Phys. B570, 455 (2000).

[19] M. Fujii and K. Hamaguchi, Higgsino and wino dark matter from Q ball decay, Phys. Lett. B 525, 143 (2002).

[20] G. B. Gelmini and P. Gondolo, Neutralino with the right cold dark matter abundance in (almost) any supersymmetric model, Phys. Rev. D 74, 023510 (2006).

[21] R. Kitano, H. Murayama, and M. Ratz, Unified origin of baryons and dark matter, Phys. Lett. B 669, 145 (2008).

[22] B. Dutta, L. Leblond, and K. Sinha, Mirage in the sky: Nonthermal dark matter, gravitino problem, and cosmic ray anomalies, Phys. Rev. D 80, 035014 (2009).

[23] R. Catena, N. Fornengo, A. Masiero, M. Pietroni, and F. Rosati, Dark matter relic abundance and scalar-tensor dark energy, Phys. Rev. D 70, 063519 (2004). 
[24] G. B. Gelmini and P. Gondolo, Ultracold WIMPs: Relics of nonstandard pre-BBN cosmologies, J. Cosmol. Astropart. Phys. 10 (2008) 002.

[25] A. B. Lahanas, N. E. Mavromatos, and D. V. Nanopoulos, Dilaton and off-shell (noncritical string) effects in Boltzmann equation for species abundances, PMC Phys. A 1, 2 (2007).

[26] M. T. Meehan and I. B. Whittingham, Dark matter relic density in scalar-tensor gravity revisited, J. Cosmol. Astropart. Phys. 12 (2015) 011.

[27] B. Dutta, E. Jimenez, and I. Zavala, Dark matter relics and the expansion rate in scalar-tensor theories, J. Cosmol. Astropart. Phys. 06 (2017) 032.

[28] H. Baer, V. Barger, P. Huang, D. Mickelson, M. PadeffkeKirkland, and X. Tata, Natural SUSY with a bino- or wino-like LSP, Phys. Rev. D 91, 075005 (2015).

[29] L. Aparicio, M. Cicoli, B. Dutta, S. Krippendorf, A. Maharana, F. Muia, and F. Quevedo, Nonthermal CMSSM with a 125 GeV Higgs, J. High Energy Phys. 05 (2015) 098.

[30] A. G. Delannoy et al., Probing Dark Matter at the LHC using Vector Boson Fusion Processes, Phys. Rev. Lett. 111, 061801 (2013).

[31] P. Poulose, S. Sahoo, and K. Sridhar, Exploring the inert doublet model through the dijet plus missing transverse energy channel at the LHC, Phys. Lett. B 765, 300 (2017).

[32] V. Khachatryan et al. (CMS Collaboration), Search for Dark Matter and Supersymmetry with a Compressed Mass Spectrum in the Vector Boson Fusion Topology in ProtonProton Collisions at $\sqrt{s}=8 \mathrm{TeV}$, Phys. Rev. Lett. 118, 021802 (2017).
[33] G. Aad et al. (ATLAS Collaboration), Search for invisible decays of a Higgs boson using vector-boson fusion in $p p$ collisions at $\sqrt{s}=8 \mathrm{TeV}$ with the ATLAS detector, J. High Energy Phys. 01 (2016) 172.

[34] J. Alwall, R. Frederix, S. Frixione, V. Hirschi, F. Maltoni, O. Mattelaer, H. S. Shao, T. Stelzer, P. Torrielli, and M. Zaro, The automated computation of tree-level and next-to-leading order differential cross sections, and their matching to parton shower simulations, J. High Energy Phys. 07 (2014) 079.

[35] T. Sjöstrand, S. Ask, J. R. Christiansen, R. Corke, N. Desai, P. Ilten, S. Mrenna, S. Prestel, C. O. Rasmussen, and P.Z. Skands, An introduction to Pythia 8.2, Comput. Phys. Commun. 191, 159 (2015).

[36] J. de Favereau, C. Delaere, P. Demin, A. Giammanco, V. Lemaître, A. Mertens, and M. Selvaggi (DELPHES Collaboration 3), DELPHES 3, A modular framework for fast simulation of a generic collider experiment, J. High Energy Phys. 02 (2014) 057.

[37] M. Cacciari, G. P. Salam, and G. Soyez, FastJet user manual, Eur. Phys. J. C 72, 1896 (2012).

[38] A. Goudelis, B. Herrmann, and O. Stål, Dark matter in the inert doublet model after the discovery of a Higgs-like boson at the LHC, J. High Energy Phys. 09 (2013) 106.

[39] A. Alloul, N. D. Christensen, C. Degrande, C. Duhr, and B. Fuks, FeynRules 2.0-A complete toolbox for tree-level phenomenology, Comput. Phys. Commun. 185, 2250 (2014).

[40] M. Aaboud et al. (ATLAS Collaboration), Search for new phenomena in final states with an energetic jet and large missing transverse momentum in $p p$ collisions at $13 \mathrm{TeV}$ using the ATLAS detector, Phys. Rev. D 94, 032005 (2016). 